\title{
Percutaneous Cystolitholapaxy Using the LithoClast Trilogy for Multiple Bladder Stones
}

\author{
Matthew H.V. Byrne, MBBS, MRes, ${ }^{1}$ Laurian Dragos, MD, ${ }^{1}$ Andrew Winterbottom, MBBCh, ${ }^{2}$ \\ Kasra Saeb-Parsy, MBBS, BSc, ${ }^{1}$ and Oliver J. Wiseman, MBBChir, MA ${ }^{1}$
}

\begin{abstract}
Background: Multiple large bladder calculi are traditionally managed through open cystolithotomy or transurethrally in patients who have an open bladder neck. Open procedures are technically challenging in patients who may have had multiple previous open surgeries, and may be associated with significant morbidity in patients with a high comorbid burden. Therefore, a percutaneous approach to such stones has been used especially when there is a closed bladder neck, and has been shown to be effective. There are a number of available devices for breaking stones in these approaches, the newest of which is the LithoClast Trilogy ${ }^{\mathrm{TM}}$ (EMS, Nyon, Switzerland) device, a probe that provides ultrasonic and mechanical calculi fragmentation and suction in a single instrument.

Case Presentation: We describe the first reported case of percutaneous cystolitholapaxy using the LithoClast Trilogy device in a 41-year-old woman with spina bifida, and multiple large bladder calculi with a history of ileocystoplasty and Mitrofanoff formation, and a bladder neck closure for neuropathic bladder. The calculi measured 31 and $25 \mathrm{~mm}$, and had a volume of 19.6 and $7.9 \mathrm{~cm}^{3}$ and average HU of 408 and 462, respectively. The calculi were composed of $37 \%$ calcium phosphate and $63 \%$ magnesium ammonium phosphate.

Conclusion: We demonstrate that this approach can be used as a viable alternative to open surgery, which is of particular importance for complex patients who have undergone multiple previous open operations, and who may have a high comorbid burden.
\end{abstract}

Keywords: bladder stones, cystolitholapaxy, lithoclast, ileocystoplasty, Mitrofanoff

\section{Introduction}

$\mathbf{M}$

ULTIPLE LARGE BLADDER calculi are most commonly managed through open cystolithotomy. ${ }^{1}$ They may be managed transurethrally in patients who have an open bladder neck, either using a stone punch, holmium laser, or, as most recently described, using the LithoClast Trilogy ${ }^{\mathrm{TM}}{ }^{2}$ However, open procedures are technically challenging in patients who may have had multiple previous open surgeries, and may be associated with significant morbidity in patients with a high comorbid burden. Therefore, a percutaneous approach to such stones has been used in such situations, and has been shown to be effective. ${ }^{3}$ The LithoClast Trilogy (EMS, Nyon, Switzerland) device is a probe that provides ultrasonic and mechanical calculi fragmentation and suction in a single instrument. ${ }^{4}$

We describe the first reported case of percutaneous cystolitholapaxy using the LithoClast Trilogy device, in a patient with a background of ileocystoplasty and Mitrofanoff and bladder neck closure.

\section{Case Presentation}

A 41-year-old woman presented with a 7-month history of recurrent urinary tract infections and difficult selfcatherization on a background of spina bifida, for which she had undergone a clam ileocystoplasty and Mitrofanoff with bladder neck closure. She also had had a previous hysterectomy, a ventriculoperitoneal shunt, and flaccid paraplegia. Computed tomography was performed to assess stone burden and surrounding anatomy, especially to ensure no bowel was between bladder and skin. It showed two large bladder calculi measuring 31 and $25 \mathrm{~mm}$, and had a volume of 19.6 and $7.9 \mathrm{~cm}^{3}$ (average HU of 408 and 462, respectively, Fig. 1 and Fig. 2) and only a small window of interposing bowel inferiorly. In these cases, cystolitholapaxy through the Mitrofanoff using a flexible ureteroscope and laser is often effective at breaking the stone, but the fragments will fail to drain. A decision was made to proceed percutaneously.

The bladder was punctured under ultrasound guidance and a guidewire was inserted into the bladder (Fig. 3). The tract

\footnotetext{
${ }^{1}$ Department of Urology, Addenbrooke's Hospital, Cambridge, United Kingdom.

${ }^{2}$ Department of Interventional Radiology, Addenbrooke's Hospital, Cambridge, United Kingdom.
} 

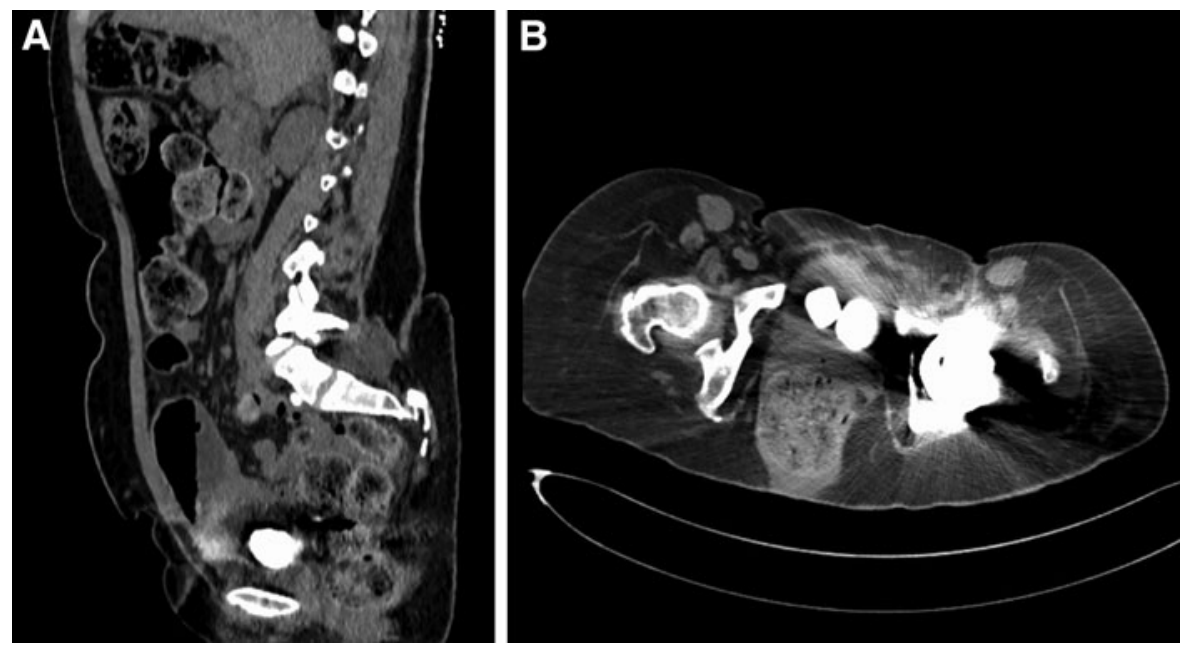

FIG. 1. Computed tomography showing multiple large bladder stones in the (A) sagittal and (B) coronal plane.

was dilated to $12 \mathrm{~F}$ and a Nephromax ${ }^{\mathrm{TM}} 30 \mathrm{~F}$ balloon was introduced to create the track. A nephroscope was used to identify the calculi and the LithoClast Trilogy device used to remove all calculi. The total operation time lasted 2 hours 28 minutes and the fragmentation time was 40 minutes and 9 seconds. Ultrasound power was $60 \%$, suction power $100 \%$, impact power $80 \%$, and impact frequency $12 \mathrm{~Hz}$. The skin was closed and catheters were inserted into the Mitrofanoff and suprapubically.

The patient was discharged the same day, and was treated with co-amoxiclav for 5 days. The calculi weighed $29 \mathrm{mg}$ and were $37 \%$ calcium phosphate and $63 \%$ magnesium ammonium phosphate.

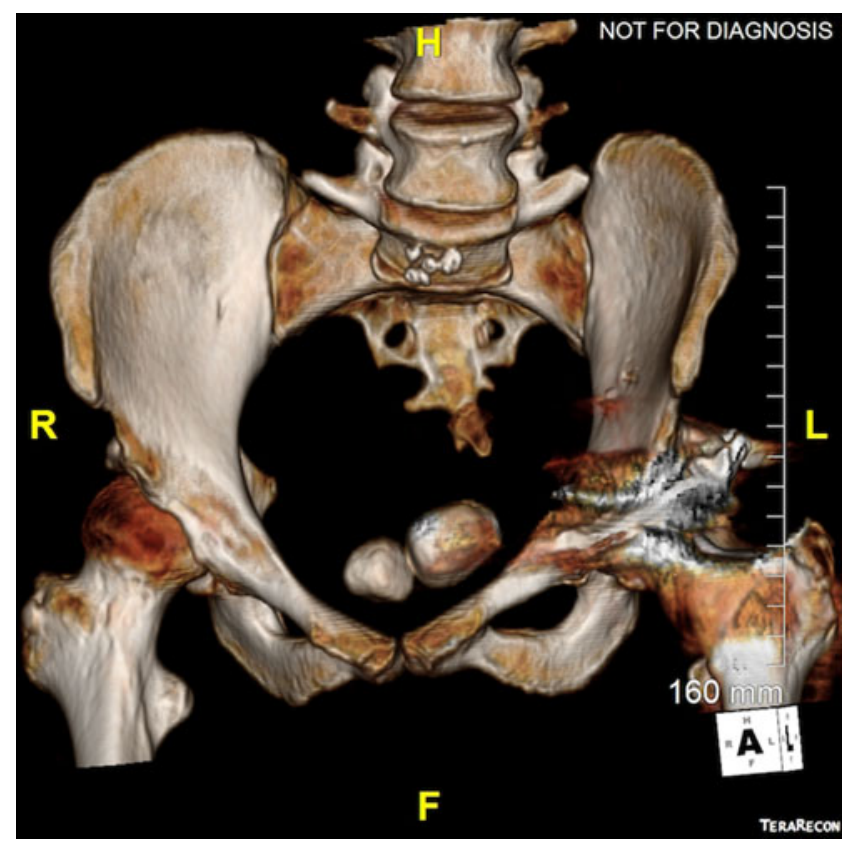

FIG. 2. Three-dimensional rendering of the computed tomography scan showing multiple bladder stones.

\section{Conclusion}

This is the first reported case of percutaneous cystolitholapaxy using the LithoClast Trilogy device. We demonstrate that this can be used as an effective fragmentation and retrieval device for patients undergoing percutaneous cystolitholapaxy.
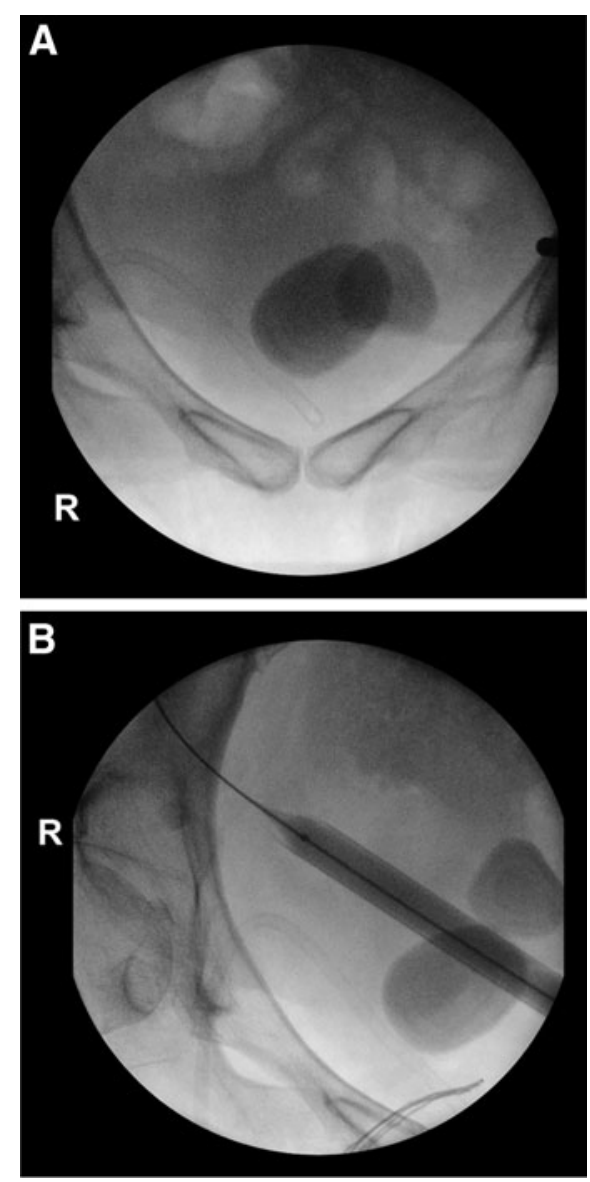

FIG. 3. Intraoperative fluoroscopy showing (A) large bladder stones and (B) the percutaneous approach. 


\section{Authors' Contributions}

M.H.V.B. and O.J.W. were responsible for writing the first draft. L.D., A.W., K.S.P., and O.J.W. were responsible for care of the patient. All authors were responsible for revisions. O.J.W. was responsible for supervision.

\section{Disclosure Statement}

O.J.W. is a consultant, education, research EMS; consultant, education Boston Scientific; and consultant, education, research Coloplast. Other authors have no conflicts of interest.

\section{Funding Information}

No funding was received for this article.

\section{References}

1. Papatsoris AG, Varkarakis I, Dellis A, Deliveliotis C. Bladder lithiasis: From open surgery to lithotripsy. Urol Res 2006;34:163-167.

2. Sninsky BC, Flamiatos JF, Nakada SY. The end of "Cutting for Stone"? Using the LithoClast Trilogy for cystolitholapaxy on a $4 \mathrm{~cm}$ bladder stone per urethra. Urol Case Rep 2019;26:100964.

3. Abdelhafez MF, Bedke J, Amend B, et al. Minimally invasive percutaneous nephrolitholapaxy (PCNL) as an effective and safe procedure for large renal stones. BJU Int 2012;110: E1022-E1026.
4. Sabnis RB, Balaji SS, Sonawane PL, et al. EMS LithoClast Trilogy $^{\mathrm{TM}}$ : An effective single-probe dual-energy lithotripter for mini and standard PCNL. World J Urol 2019:1-8; DOI: 10.1007/s00345-019-02843-2.

Address correspondence to: Matthew H.V. Byrne, MBBS, MRes Department of Urology Addenbrooke's Hospital Hills Road Cambridge CB2 OQQ United Kingdom

E-mail: mhvbyrne@doctors.org.uk

\section{Abbreviations Used \\ $\mathrm{HU}=$ Hounsfield units}

Cite this article as: Byrne MHV, Dragos L, Winterbottom A, Saeb-Parsy K, Wiseman OJ (2020) Percutaneous cystolitholapaxy using the LithoClast Trilogy for multiple bladder stones, Journal of Endourology Case Reports 6:3, 118-120, DOI: 10.1089/ cren.2019.0143. 